Einführung zum Thema

coloproctology 2019 · 41:160-161

https://doi.org/10.1007/s00053-019-0344-2

(c) Springer Medizin Verlag GmbH, ein Teil von Springer Nature 2019

Die Nachbehandlung bei proktologischen Eingriffen ist ein kritischer Bestandteil für den Erfolg der Therapie. Operationen im analen Bereich sind für die Patienten immer eine physische und psychische Belastung, verursachen sie doch meistens deutliche Schmerzen, bedürfen der intensiven Zuwendung (Wundpflege), und die Angst vor der Inkontinenz ist immer dabei ...

Für uns behandelnde Koloproktologen verursachten Rechtsstreitigkeiten im Hinblick auf die korrekte Wundversorgung in den vergangenen Jahren Unsicherheiten: Als Grundlage der Unsicherheit stand die S1-Leitlinie der AWMF für die Behandlung von chronischen Wunden, die eine Versorgung mit sterilen Spüllösungen vorsieht [1]. Hier sei explizit darauf hingewiesen, dass in den Empfehlungen kein Wort über Wunden im analen Bereich steht, und diese nur interpoliert wurden. Die über viele Jahrzehnte erfolgreich durchgeführte und "good clinical practice“ des Ausduschens mit Leitungswasser in Trinkwasserqualität, das in Deutschland flächendeckend zur Verfügung steht, kam in Verruf.

Nun haben der Berufsverband der Coloproktologen Deutschlands (BDC) und der Deutschen Gesellschaft für Koloproktologie (DGK) mit den Leitfaden und Empfehlungen für die Hygiene in der Koloproktologie Teil 1 und 2 [2, 3] eine klare Grundlage für die Empfehlungen für die Wundbehandlung nach proktologischen Operationen gelegt und die Reinigung mit Leitungswasser von Trinkwasserqualität gemäß der Trinkwasserverordnung als klare Option gewertet. Ausgenommen bleiben natürlich primär heilende Wunden und steril zu

\author{
Volker Kahlke ${ }^{1,2}$ \\ ${ }^{1}$ Proktologische Praxis Kiel, Abteilung Proktologische Chirurgie, Park-Klinik, Kiel, Deutschland \\ ${ }^{2}$ Medizinische Fakultät, Christian-Albrechts-Universität zu Kiel, Kiel, Deutschland
}

\title{
Nachbehandlung bei proktologischen Eingriffen
}

versorgende Wunden. Diese Regelungen können in den Hygieneplänen der einzelnen Einrichtungen so festgelegt werden.

\section{》) Proktologische Operationen mit sekundärer Wundheilung haben ein geringes SSI-Risiko}

Den Weg für dieses Vorgehen endgültig freigemacht haben die Empfehlungen zur Prävention postoperativer Wundinfektionen der Kommission für Krankenhaushygiene und Infektionsprävention (KRINKO) des Robert-Koch-Instituts (RKI; [4]). Auch hier werden proktologische Operationen nicht explizit erwähnt, und die Empfehlungen zielen vornehmlich auf primäre Wunden ab. Allerdings wird im letzten Kapitel „Operationen mit geringem (Surgical Site Infection) SSIRisiko" festgelegt, dass die Entscheidung, was Operationen mit geringem SSI-Risiko sind, durch den lokalen chirurgischen Fachvertreter und den beratenden Hygieniker getroffen wird. Unzweifelhaft stellen proktologische Operationen mit sekundärer Wundheilung grundsätzlich Operationen mit geringem SSI-Risiko dar.

Somit ist die Reinigung der Analregion nach proktologischen Operationen gemäß dem eigenen Hygieneplan bzw. bisher üblichen Standard beizubehalten - im Zweifelsfall, bis eine endgültige Klärung dieser Frage erfolgt bzw. eine klärende Empfehlung durch die KRINKO vorliegt. Dieses schließt eine Reinigung der Analregion postoperativ mit Leitungswasser in Trinkwasserqualität ein.

Die Autoren der einzelnen Kapitel für die Nachbehandlung bei proktologi- schen Eingriffen stellen nun einerseits die Vorgehensweise bei klassischen offenen und damit der sekundären Wundheilung folgenden Operationen dar (Beitrag von T. Jacobi). Hier wird klar die Wundversorgung/Analhygiene mit Leitungswasser in Trinkwasserqualität präferiert. Auch wird klargestellt, dass die Grundlage für die gute Wundheilung in der Operation liegt (z. B. ausreichendes Drainagedreieck).

\section{》) Stuhlregulierung und Schmerztherapie sind die Grundpfeiler für eine gute Nachbehandlung}

Bei den geschlossenen Verfahren entwickelten G. Kolbert und M. Stoll eine klare Systematik für die Versorgung in Abhängigkeit der Lage der Wunde (anal vs. rektal) bzw. in Abhängigkeit der Tiefe der Präparationsebene (mukosal/ submukosal vs. muskulär). Ferner weisen sie darauf hin, dass bei einigen Operationen die lange verschollen geglaubte orale Darmlavage wieder zu tragen kommt. Auch dient hier ggf. eine Nahrungskarenz und langsamer Kostaufbau dem Schutz der frischen Naht. Abgerundet wird die postoperative Versorgung durch die supportive Therapie im Beitrag von T. Laubert und J. Jongen: Die Stuhlregulierung einerseits und die Schmerztherapie andererseits sind die Grundpfeiler für eine gute Nachbehandlung bei proktologischen Eingriffen, damit der Patient die Operation nicht in schlechter Erinnerung behält. Hier werden anhand der neuesten Literatur die möglichen suppor- 
tiven Maßnahmen umfassend erläutert und übersichtlich zusammengefasst.

Der Sinus pilonidalis als eigene Entität hat ein eigenes umfangreiches Kapitel erhalten, und auch hier stellen die Autoren A. Ommer, D. Doll und I. Iesalnieks bei den offenen Verfahren die Wundversorgung/Analhygiene mit Leitungswasser in Trinkwasserqualität in den Vordergrund.

Die nachgestellten Artikel sind geeignet, die Nachsorge nach proktologischen Operationen klar und strukturiert zu erfassen. Gleichzeitig werden die aktuelle Literatur und Leitlinien kritisch und umfangreich beleuchtet.

\section{Korrespondenzadresse}

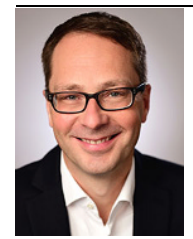

Volker Kahlke

Proktologische Praxis Kiel, Abteilung Proktologische

Chirurgie, Park-Klinik

Beselerallee 67, 24105 Kiel,

Deutschland

info@proktologie-kiel.de

Interessenkonflikt. V. Kahlke gibt an, dass kein Interessenkonflikt besteht.

\section{Literatur}

1. Leitlinie AWMF Anforderungen der Hygiene bei chronischen und sekundär heilenden Wunden. https://www.awmf.org/.../029-042l_S1_ Chronische_Wunden_Hygieneanforderungen. Zugegriffen: 15.01.2019

2. Tabori $E$, Weißgerber $P$, Berg $E$ et al (2017) Leitfaden und Empfehlungen für die Hygiene in der Koloproktologie Teil 1. Coloproctology 39:111-120

3. Tabori E, Weißgerber P, Berg E et al (2017) Leitfaden und Empfehlungen für die Hygiene in der Koloproktologie Teil 2. Coloproctology 39:205-220

4. Kommission für Krankenhaushygiene und Infektionsprävention, RKI (2018) Prävention postoperativer Wundinfektionen. Bundesgesundheitsblatt 61:448-473

\section{Springer Medizin}

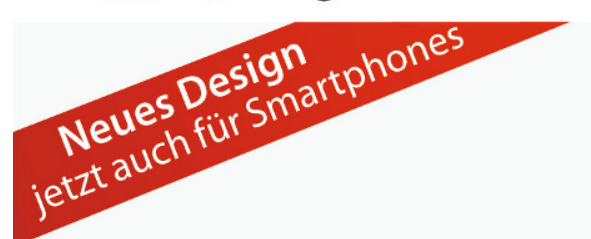

e.Curriculum Innere Medizin

\section{Clostridium difficile}

\section{E-Learning}

- Nach der aktuellen Leitlinie

- Fallbasiertes E-Learning

- Zertifiziert mit 3 Punkten

- Exklusiv für DGIM-Mitglieder und e.Med-Abonnenten

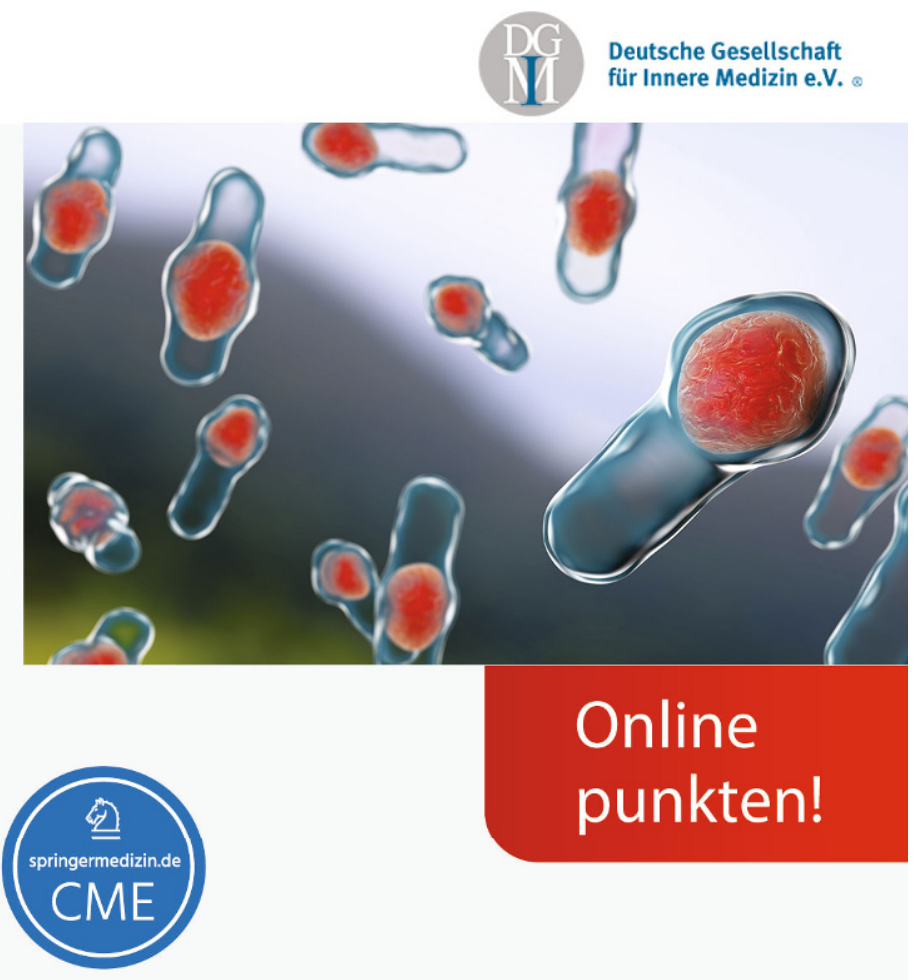

Deutsche Gesellschaft für Innere Medizin e.V. ifficile 